\title{
CORRIGENDUM
}

\section{LEARNING TO SIGNAL WITH PROBE AND ADJUST - CORRIGENDUM}

\section{BRIAN SKYRMS}

doi: IO.IOI7/epi.20I2.5, published online by Cambridge University Press I I July 20 I2.

The author wishes to make the following correction.

In 'Learning to Signal with Probe and Adjust', I said: 'Note that this system state enables us to calculate the pay-offs that they got last time they did something' (I42). I should have said '...this system state constrains the pay-offs that they got last time they did something to an extent sufficient to establish that signalling systems are the only absorbing states, and that there is a positive path from any state to a signalling system'.

The point is that when the sender pools, pay-offs last time may be underdetermined. We are dealing with a random, time-inhomogeneous Markov chain rather than a timehomogeneous chain. Nevertheless, the proof that Probe and Adjust learns to signal with probability one proceeds just as before.

Given a positive probability path from each state to an absorbing state, there is a maximum path length, $n$, and a minimum path probability, $e$. Starting from any state, the probability of not being absorbed after $n$ probes is at most (I-e). After $\mathrm{m}^{* \mathrm{n}}$ probes, the probability of not being absorbed is $(\mathrm{I}-\mathrm{e})^{\wedge} \mathrm{n}$. In the limit, the probability of not being absorbed is 0 .

\section{REFERENCE}

Skyrms, Brian. 20I2. 'Learning To Signal With Probe And Adjust.' Episteme, 9: I39-50. doi:IO.IOI7/epi.2OI2.5. 